\title{
Evaluation of school oral health education program- A review
}

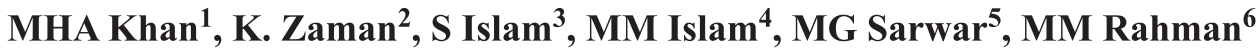

\begin{abstract}
The objectives of this review were to examine the various methods of school oral health instruction process and to identify success factors for oral health promotion along with the development of a future framework of action for Oral Health Promotion. In this review, the members of the working groups performed the systematic literature search, screening process, rating of the evidence, assessment of the evidence and presentation of the contents of the evidence to the reader. In this study we performed literature searched published between 1990 to 2013. Using database including MEDLINE, PUBMED, HINARI, Google and yahoo health. A total of 31 articles were retrieved and 15 of them were selected for review.
\end{abstract}

Inclusion and exclusion criteria were chosen by working group such as studies reported any oral health promotion intervention in school were eligible for inclusion, Study conducted in the school for adult education or handicapped or disables were excluded, oral health education for adult were excluded. In this review the working group tried to synthesize the qualitative and quantitative findings from the selected article. All studies were interventiona, the main intervention was oral health education in variable format along with other activities like fissure sealant, application of fluoride, distribution of tooth brush and toothpaste. In oral health education, presentation materials such as poster, tooth brushing model, life model and multimedia presentation were used. The investigators in five studies distributed tooth brush, tooth paste, tooth brushing calendar etc.

Maximum investigators concluded that school oral health education is more or less effective in all situations, the summary of this review confirmed the hypothesis that there would be better and more homogenous oral health in schools where comprehensive oral health activities were developed compared with non-supportive schools. School oral health improvement activities has a great impact for the improvement of oral health among the children. The efficacy of oral health education is well-established and it was found that Oral health education in the school decreased the development of new dental diseases among school children. Majority of students had an adequate level of knowledge on oral health but low level of oral health practices. Reinforcement and encourage to maintain the oral health will be more effective for the long term sustainable oral health.

Keywords: Dental health education, school oral health, school tooth brushing instruction.

\section{Introduction}

Schools provide an important setting for promoting the oral health. Health promotion message can be reinforced throughout the most influential stages of children's life, enabling them to develop lifelong sustainable attitudes and skills.

1. Dr. Md. Haider Ali Khan, Assistant Professor, Department of Dental Public Health, Dhaka Dental College \& Hospital, Mirpur, Dhaka

2. Prof. Dr. Khursiduzzaman, Professor and Head of the Department, Department of Dentistry, Dinazpur Medical College \& Hospital, Dinazpur

3. Dr. Shariful Islam, Associate Professor, OSD, DGHS, Mohakhali, Dhaka

4. Dr. Meftaul Islam, Associate Professor, MARKS Dental College \& Hospital, Mirpur, Dhaka

5. Dr. Md. Golam Sarwar, Assistant Professor, Department of Dental Public Health, Dhaka Dental College \& Hospital, Mirpur, Dhaka

6. Dr. Md. Mostafizur Rahman, BDS, Consultant, Joyes Dental Care, Dhaka

Address of Correspondence:

Dr. Md. Haider Ali Khan, Assistant Professor, Department of Dental Public Health, Dhaka Dental College \& Hospital, Mirpur, Dhaka

E mail: haiderkhan3@hotmail.com
Poor oral health can have a detrimental effect on children's quality of life, their performance at school and their success in later life. Other than that the spillover effect of school oral health instruction, it is also beneficial for other school children, their families, school teachers and the community as a whole ${ }^{1}$.

Oral health is fundamental to general health and well-being. A healthy mouth enables an individual's to speak, eat and socialize without experiencing active disease, discomfort or embarrassment. Children who suffer from poor oral health are 12 times more likely to have restricted activity than those who do not ${ }^{2}$. More than 50 million school hours are lost annually because of oral health problems which affect children's performance at school and success in later life. ${ }^{3}$ For many years, schools have been recognized as important settings for health education. Traditionally, health education focused on providing information or developing skills and attitudes to help individuals make healthy choices about lifestyle or behavior.

In the 1980s, the World Health Organization (WHO) shifted focus from the behavior of individuals to the development of healthy 'settings'. This resulted in the eco-holistic approach of health promotion as defined in the Ottawa Charter (WHO, 1986). 
The principles of the Ottawa Charter were applied to the school setting, resulting in the concept of the 'health promoting school'. Within this approach, focus is increasingly shifting from programs that are specific to certain health aspects, to those that have a holistic approach to health promotion. ${ }^{4}$

Over the last decade, health promoting schools have been implemented in many countries and regional networks have been established. Oral health care instruction programs and school healthcare systems were established in the USA in the 1940s and in Japan in the $1960 \mathrm{~s}^{5}$ School oral health activities has a favorable impact on the school environment and the health of school communities. Research has shown that adolescent health is influenced by several interlinked factors ${ }^{6}$

School has the potential link with children and adolescents, families and community members. Children may also be equipped with personal skills that enable them to make healthy decisions, adopt healthy lifestyles. Schools can also serve as important channels to communities. School personnel can target health promotion activities to homes and communities. Students can pass health-promotion messages on to family members. And schools can take the lead in advocating for viewing oral health as part of general health. Schools can provide supportive environments for promoting oral health. A safe physical environment in the playground and throughout a school can help reduce the risk of dental trauma. ${ }^{7}$

The consequences of oral disease are not only the physical but they are also economic, social and psychological. ${ }^{8}$ Dental caries is the most prevalent dental affliction in humans. Despite creditable scientific advances and the fact that caries is preventable, the disease continues to be a major public health problem predominantly affecting children. Dental caries is often responsible for the absenteeism from school and loss of working hours. The lack of availability of dental care, postponement of treatment due to cost considerations, and under utilization of available facilities not only results in aggravation of the disease but also enhances the cost of treatment and care. Worldwide schools offer an efficient and effective way to reach children and, through them, families and community members.

School age is an influential stage in people's lives, a time when lifelong sustainable oral health - related behaviors, as well as beliefs and attitudes, are being developed. 9
Children are particularly receptive to health messages during this period and the earlier the good habits are established, the longer lasting the impact.

The most common oral problems, caries and periodontal diseases, are bacterial in origin, exacerbated by dietary sugars, ineffective plaque removal, and less than optimal fluoride availability. Dental caries afflicts humans of all ages and in all regions of the world and is a disease of the complex interplay of social, behavioral, cultural, dietary, and biological risk factors that are associated with its initiation and progression. Regardless of the fact that caries is preventable, its prevalence is high and still increasing in some developing countries, especially among urban children, while its decline has been reported in many industrialized countries during the last three decades. ${ }^{10}$ The increase seems to be mainly a consequence of increasing consumption of sugar-containing snacks and soft drinks due to urbanization, combined with insufficient use of fluoride and inadequate oral hygiene. ${ }^{11,12}$

Dental diseases are not directly life-threatening, but have a detrimental effect on quality of life: having an impact on normal social roles, self-esteem, nutrition, communication, and general health, and causing pain, discomfort, and loss of function. At a society level, treating dental diseases is very costly for health care systems. The costs account for $5 \%$ to $10 \%$ of total health care expenditure in industrialized countries, exceeding that for treating cardiovascular disease, cancer, and osteoporosis. Because in developing countries, the cost of traditional curative care of dental diseases would probably exceed the available resources for health care, preventive strategies are clearly more affordable and sustainable. ${ }^{13}$

\section{Materials and Methods}

At first a workgroup is formed by the combination of the teachers and researchers who are related with school oral health activities. The working team performed the systematic literature search, screening process, application of inclusion and exclusion criteria, rating of the evidence, assessment of the evidence and presentation of the contents of the evidence to the reader. In this study we performed literature search published between 1990 to 2013 . 
Using five database including MEDLINE, PUBMED, HINARI, Google and yahoo health. Only studies that focused school oral hygiene instruction process along with or without other activities were included in this review process. A total of 31 articles were retrieved and 15 of them were selected for review. All protocols were cited on the World Wide Web (www) by applying the key words; school oral health activities, oral hygiene instruction, school tooth brushing instruction etc.

The reference lists of retrieved papers were examined for additional studies, Papers only published in English were included in this review. Multiple reports from the same study only contributed once to the review, whereas papers which used a variety of outcome measures were included in each outcome category.

\section{Research questions}

We addressed the following questions;

1) What are the methods used in the school oral hygiene instruction program?

2) Which one is the most effective method of oral hygiene instruction?

3) Is school oral hygiene instruction really effective?

We systematically reported the relevant scientific information for each question.

Objectives: the main objective of the review is

1. To examine the various methods of school oral health instruction process

2. To gather information on the structures, processes and outcomes of oral health promotion/education initiatives and resources

3. To identify performance indicators for oral health instruction

4. To identify success factors for oral health promotion

5. To develop a future framework for action for Oral Health Promotion/Education

\section{Inclusion and exclusion criteria of article}

Studies reported any oral health promotion intervention in school were eligible for inclusion, The following interventions were included in the review: tooth brushing programmes in schools; education of the parents to improving children's dental health; group and individual education sessions, school oral health activity along with prophylaxis; fluoride tablets, gels, drops and rinses were included in this study. The studies performed among the elementary schools were included, study conducted in the school for adult education or handicapped or disable were excluded, oral health education for adult was excluded.

\section{Results and discussion}

In this review the working group tried to synthesize the qualitative and quantitative findings from the selected articles. Meta analysis of the quantitative findings was poor due to lack of quantitative data in the maximum articles. All studies were interventional; the main intervention was oral health education in variable format along with other activities like fissure sealant, application of fluoride, distribution of tooth brush and toothpaste uses of multimedia presentation, presentation materials such as poster, tooth brushing models etc. The investigators in five studies distributed tooth brush, tooth paste, tooth brushing calendar etc.

In two studies conducted by Haleem et $\mathrm{al}^{14}$ (2012) and Mellanby et $\mathrm{al}^{15}(2000)$ oral health educator was categorized by teacher-led, dentist- led, peer-led and self learning strategy and compared the effectiveness among the educators group. Although the three educator-led strategies had a modest effect on the oral health improvement but the results provided some evidence to show that the peer-led strategy may provide a feasible and almost equally effective alternative to the traditional dentist-led strategy of oral health education. The peer-led and dentist-led methods of oral health education were shown to perform better than the self-learning strategy in both studies. One significant finding of the their study was that the peer-led strategy was almost as effective as the dentist-led strategy and comparatively more effective than the teacher-led and self- learning strategies in improving preventive oral health behavior about gingivitis, dental caries. The improvement in oral health knowledge resulting from dental health education interventions has been a consistent finding in the dental literature. The investigator concluded that the three educator-led strategies produced about a six fold increase in knowledge as compared to the control group

To make the oral health education more attractive and interesting some investigators conducted a tooth brushing session at school. Re- enforcement or follow up visit to the school varies from study to study, some planned for monthly, quarterly or half yearly follow up strategy.

Stella ${ }^{16}$ and Stylianos ${ }^{17}$ concluded that there were two great changes after implementing oral health education program that is Positive changes were observed in a number of the reported dental health knowledge and behaviors have changed after school oral health education. 
Friel ${ }^{18}$ compared oral health education program with television oral health campaign, he concluded, school oral health education and mass media campaign both are effective but school oral health program gives extra benefits to both way communication, furthermore it facilitates access to every child

Kaketa ${ }^{19}$ reported the spillover effect of school oral health education that gives extra benefits to other children, school teachers, and overall to the related community. The community can achieve marked improvement and remains very positive about the program. Other than that, school oral health promotion activities increasing 'life skills', such as problem-solving, communication, decision-making and coping with emotions, which may improve many aspects of physical, mental and social health.

Bodner ${ }^{20}$ compared the effectiveness of school oral health activities along with application of sealant in the context of measuring DMFT, DMFT score was compared at follow up between second graders who had received oral health education with sealant and those who did not receive. The result of the analysis showed that the second graders who had not received oral health instruction had higher DMFT score than who received. The average percent of first molars decayed, missing or filled was $3.2 \%$ for the intervention group and $6.7 \%$ for the non intervention group. Among the children who received oral health education also showed the reducing trend of development of new caries

Peixoto ${ }^{21}$ done a component analysis by considering the mean overall score as a cut-off point, a dichotomous variable was constructed, classifying schools as either supportive or non-supportive. A supportive school was one where the development of health promotion components was above the mean, while a non supportive school was the reverse, i.e. below the mean. The purpose was to investigate for whom, and for what type of school, was the impact on children's oral health status the best. Significant relationships were found between the outcomes and some singular components representing the dimensions of health promotion in the schools. A moderately positive correlation was found between the different components, a higher percentage of caries free children were related to the supportive school. The overall outcomes analysis and estimates were calculated separately for supportive and non-supportive schools.
The crude summary measure, children in supportive schools, where health promotion activities had been developed, had better oral health than children in non-supportive schools in deprived areas. More inequalities in oral health were observed in non supportive than in supportive schools.

Horold ${ }^{22}$ and Stella ${ }^{16}$ suggested that the efficacy of school oral health education is well-established. It was found that Oral health education in the school decreased the development of new dental diseases among children. However, the program was unable to improve children's oral health at the population level without performing it in school. Oral health education program must be designed for and directed to a specific audience such as school children. However, there are good arguments for directing programs toward school children, and also toward parents, so that they can mutually reinforce each other in the learning process.

Yonemitsu ${ }^{23}$ and Stylianos ${ }^{17}$ conducted a study to evaluate the health activities at school which consisted of tooth brushing instruction in class, dental education for the schoolchildren and their guardians, utilization of fluoride mouth rinsing (once a week), and, by following a model school of caries prevention program. The oral health status of this primary school children improved significantly compared with those of the school children who followed the routine school dental health activities only. The results also showed that the oral health behavior of interventional primary schoolchildren also improved.

Rong et $\mathrm{al}^{24}$ conducted a study in China at the evaluation, a significantly higher percentage of children in the test group than in the control group reported brushing their teeth twice a day after receiving oral hygiene instruction. Parents of children in the test group had better oral health knowledge and attitude than the parents of children in the control group. Peterson ${ }^{25}$ concluded oral health education program was effective in establishing good oral health habits among preschool children and in increasing oral health knowledge of their parents, in conjunction with supervised daily tooth brushing with fluoridated toothpaste, which could reduce the development of new dental caries in preschool children in China.

Kay $^{26}$ suggested that short follow-up showed significant improvements in plaque levels, whilst studies with long periods of follow-up suggested that instruction and education about plaque control were not effective in the long term. 
These studies indicated that knowledge levels invariably altered by the interventions. Complex and technical educative methods added little benefit, and simple provision of information was sufficient to increase knowledge levels. However, the studies that included other outcome measures also suggested that alterations in knowledge, attitudes and beliefs were not related to changes in behavior or health.

Sakuma ${ }^{27}$ did an economic evaluation by cost-effectiveness and cost-benefit analyses. Economic viability or cost- effectiveness was found in oral health activities over the treatment for the disease. It is possible that when the children will be conscious regarding their oral health, the treatment costs could be lower than actual costs of treatment at letter stage due to self realization effect .

\section{Recommendations}

Oral hygiene status shows a very high label regional variation, furthermore it also depends on the health believes, behaviors, cultures and other many factors, so it is recommended that, the region wise extensive study should be carried out to observe the effectiveness of the school oral health activities.

Along with school oral health improvement activities, oral health knowledge and practices should be taught to school students. The oral health teaching manual should be used by including newer concepts of oral health care, daily oral health practice and maintenance along with mouth healthy diet practice.

There should be some degree of agreement between the administrative and teaching staff on a common educational philosophy in regard to child oral health improvement. The administrative staff should be willing to assume a leadership role in developing and improving the curriculum. And finally, the teachers and administrators should not be reluctant to implementing the programs for dental health education.

There is a need to develop clearly defined, valid, feasible and utilizable indicators to evaluate school oral health education process, output and outcome. Indicators may differ between schools, networks or regions, depending on the intervention framework adopted and theoretical positions.

\section{Conclusions}

The summary of this review confirmed the hypothesis that there should be better and more homogenous oral health in schools where comprehensive oral health activities were developed compared with non-supportive schools.
School oral health improvement activities have a great impact for the improvement of oral health among the children. Majority of students had an adequate level of knowledge on oral health but low level of oral health practices. Reinforcement and encouragement to maintain the oral health will be more effective for the long term sustainable oral health.

Effectiveness of the school oral health program depends on multiple factors like culture, health behavior, food habits, economic and health environments, no particular method or technique is solely effective for overall improvement of the oral health status, it should be modified or adjusted according to the need of the society. To maximize the strengths of oral health education approach it is necessary to repeatedly discuss with the related personnel.

\section{References}

1. Rua Silveira Peixoto, Curitiba PR. Associations between health promoting schools' policies and indicators of oral health in Brazil. Health Promotion International, Vol. 18, No. 3 C Oxford University Press 2003.

2. US General Accounting offices. Oral health: dental disease is a chronic problem among low-income populations, Washington, DC: report to congressional requesters: 2000 .

3. Gift HC, Reisine ST, Larach DC. The social impact of dental problems and visits, American Journal of Public Health 1992; 82:1663-8.

4. World Health Organization: Oral health promotion: an essential element of a health-promoting school. WHO Information Series on School Health Document Eleven. Geneva: World Health Organisation; 2003.

5. Risa AOKI, Yasuiko MORITA, Khurshiduz Zaman, Zunaid Ahmed, Md. Haider Ali Khan, Hiro-o ITO, Syuichi TAKINAMI: Motivation and Technical Instruction of Tooth Brushing Instruction (TBI) to the Primary School Teachers and students.

Link:http://www16.ocn.ne.jp/ yasuhiko/JICA/JICA.ht $\mathrm{ml} \#$ LinkPub

6. Flisher, A. J., Kramer, R. A., Hoven, C. W., King, R. A.,Bird, H. R., Davies, M. et al. (2000) Risk behavior in a community sample of children and adolescents. Journal of the American Academy of Child and Adolescent Psychiatry, 39, 881-887.

7. Bertness, J, Holt K, eds. 2009. Promoting Oral Health in Schools: A Resource Guide-April 2009. Washington, DC: National Maternal and Child Oral Health Resource Center. 
8. Mariko Naito, Hidemichi yuasa, Yoshiaki Nomura, Takeo Nakayama, Nobuyuki Hamajima, and Nobuhiro Hanada. Oral health status and health-related quality of life: a systematic review. Journal of Oral science, Vol.48,No.1, 1-7,2006

9. World Health Organization. The Status of School Health. Report of the School health Working Group and the WHO Expert Committee on Comprehensive School Health Education and Promotion.1 Geneva: WHO; 1996.

10. Petersen PE. The World Oral Health Report 2003: continuous improvement of oral health in the $21 \mathrm{st}$ century-the approach of the WHO Global Oral Health Programme. Community Dent Oral Epidemiology 2003; 31(Suppl 1):3-24.

11. Sheiham A. Changing trends in dental caries. Int J Epidemiol 1984;13:142-147.

12. Cirino SM, Scantlebury S. Dental caries in developing countries. N Y State Dent J 1998;64:32-39.

13. Sheiham A. Dietary effects on dental diseases. Public Health Nutr 2001;4:569-591.

14. Abdul Haleem, Muhammad Irfanullah Siddiqui and Ayyaz Ali Khan: School-based strategies for oral health education of adolescents- a cluster randomized controlled trial, BMC Oral Health 2012, 12:54 doi:10.1186/1472-6831-12-54(led strategy)

15. Mellanby AR, Rees JB, Tripp JH: Peer-led and adult-led school health education: a critical review of available comparative research. Health Educ Res 2000, 15:533-545.

16. Stella Y.L. Kwan, Poul Erik Petersen, Cynthia M. Pine, Annerrose Borutta. Health promoting School: an opportunity for oral health promotion. Bulletin of the world health organization, 2005;83:677-685

17. Stylianos M. Piperakis, Anna Kritsi, Fotini Garagouri-Araiou and Xanthipi Gialama: A survey on the knowledge, attitude and behavior of Greek elementary School students about oral health and dental care. Pediatric Dental Journal 18(2): 167-175, 2008.

18. S. Friel, A. Hope, C. Kelleher, S. Comer and D. Sadlier:Impact evaluation of an oral health intervention amongst primary school children in Ireland: oxford journal health promotion international(2002); volume17(2), 119-126
19. Toshitaka Kaketa: Oral health in Japan, Journal of Oral health and Dental management,Volume 1 Issue 1(2002)

20. Sandy Bodner, Liz Pulos Evaluation of a School-Based Oral Health Program. Tacoma Pierce County Health Department Pierce County, Washington, May 2010

21. Rua Silveira Peixoto, Curitiba, PR Associations between health promoting schools'policies and indicators of oral health in Brazil, Health Promotion International, Vol. 18, No. 3 C Oxford University Press 2003.

22. Horold I, Applewchite, Dental Health Education in the Schools, American Journal of Public Health. VOL. 60, NO. 9. 1835-1838

23. Yonemitsu M, Kawaguchi Y, Ohara S, Hirayama Y, Sasaki Y Handa. Evaluation of school dental health activities in Hiraizumi primary school, Iwate prefecture. Department of Preventive Dentistry and Public Health, Faculty of Dentistry, Tokyo Medical and Dental University.

24. Rong WS, Bian JY, Wang WJ, Wang JD) Effectiveness of an oral health education and caries prevention program in kindergartens in China.

25. Petersen PE, Esheng Z. Dental caries and oral health behaviour situation of children, mothers and schoolteachers in Wuhan, People's Republic of China. Int Dent J 1998;48: 210-216.

26. Kay EJ, Locker D. Is dental health education effective? A systematic review of current evidence. Community Dent Oral Epidemiol 1996; 24: 231-5.

27. Shihoko Sakuma, Akihiro Yoshihara, Hideo Miyazaki and Seigo Kobayashi: Economic Evaluation of a School-based Combined Program with a Targeted Pit and Fissure Sealant and Fluoride Mouth Rinse in Japan. The Open Dentistry Journal, 2010, 4, 230-236 